Скопје, Македонија

\title{
MODIFIED BASKAKOV-KANTOROVICH OPERATORS PROVIDING A BETTER ERROR ESTIMATION
}

\author{
QIULAN QI AND GE YANG
}

\begin{abstract}
We introduce a kind of Baskakov-Kantorovich operators, which preserve the test functions 1 and $x^{2}$. This type of modification enables better error estimation on the interval $\left[\frac{\sqrt{3}}{3},+\infty\right)$ than the classic ones. Finally, a Voronovskaya-type theorem for these operators is also obtained.
\end{abstract}

\section{INTRODUCTION}

King-type approximation operators[1-7] preserving the test functions 1 and $x^{2}$, and have better approximation properties than the classical ones. Motivated by this, we introduce a kind of Baskakov- Kantorovich operators, which preserve the test functions 1 and $x^{2}$ and have better error estimation on the interval $\left[\frac{\sqrt{3}}{3},+\infty\right)$ than the classical Baskakov-Kantorovich operators.

Then, the classical Baskakov-Kantorovich operators are defined by

$$
V_{n}^{*}(f, x)=n \sum_{k=0}^{\infty} v_{n, k}(x) \int_{\frac{k}{n}}^{\frac{k+1}{n}} f(t) d t,
$$

where $v_{n, k}(x)=\left(\begin{array}{c}n+k-1 \\ k\end{array}\right) x^{k}(1+x)^{-n-k}, f \in C_{\beta}[0,+\infty):=\{f \in C[0,+\infty)$ : $|f(t)| \leq M(1+t)^{\beta}$ for some $\left.M>0, \beta>0\right\}$.

Let $f \in C_{\beta}[0,+\infty), u_{n}(x)=\frac{-1+\sqrt{n(n+1) x^{2}+\frac{2}{3}-\frac{1}{3 n}}}{n+1}, x \geq \frac{\sqrt{3}}{3}$ and $n \in N$, then we get the following modified positive linear operators:

$$
V_{n}^{* *}(f, x)=n \sum_{k=0}^{\infty} v_{n, k}\left(u_{n}(x)\right) \int_{\frac{k}{n}}^{\frac{k+1}{n}} f(t) d t
$$

We will give the moments and convergence theorem of our operators, which preserving the test functions 1 and $x^{2}$.

The main result of this paper is:

2010 Mathematics Subject Classification. 41A25, 41A36.

Key words and phrases. Baskakov-Kantorovich operators; modulus of continuity; Voronovskaya-type theorem. 
Theorem 1. Let $f \in C_{B}[0,+\infty)$, the space of all bounded functions on $[0,+\infty)$, for $x \geq \frac{\sqrt{3}}{3}, n \in N$, we have

$$
\left|V_{n}^{* *}(f, x)-f(x)\right| \leq 2 \omega\left(f, \sqrt{\frac{\varphi^{2}(x)}{n}-\frac{1}{2 n^{2}}}\right),
$$

where $\varphi^{2}(x)=x(1+x)$ for $V_{n}^{* *}$, the modulus of continuity of $f$ denoted by $\omega\left(f, \delta_{x}\right)$ for $\delta_{x}>0$, is defined to be

$$
\omega\left(f, \delta_{x}\right)=\sup _{|t-x| \leq \delta_{x}, t \in[0,+\infty)}|f(t)-f(x)| .
$$

Throughout this paper, $M$ denotes a positive constant independent of $n$ and $x$ and not necessarily the same at each occurrence.

\section{THE CONVERGENCE THEOREM OF THE MODIFIED OPERATORS}

By calculation, we can obtain the following result.

Lemma 1. For each $x \geq \frac{\sqrt{3}}{3}$, we have

(1). $V_{n}^{* *}(1, x)=1$;

(2). $V_{n}^{* *}(t, x)=\frac{\sqrt{n(n+1) x^{2}+2 / 3-\frac{1}{3 n}}}{n+1}-\frac{n-1}{2 n(n+1)}$;

(3). $V_{n}^{* *}\left(t^{2}, x\right)=x^{2}$.

By Lemma 1, it is clear that the operators $V_{n}^{* *}$ given by (1.2) preserve the test functions 1 and $x^{2}$. Then from Lemma 1, one can get the following results for moments.

Lemma 2. For each $x \geq \frac{\sqrt{3}}{3}$, we have

(1). $V_{n}^{* *}(t-x, x)=\frac{\sqrt{n(n+1) x^{2}+2 / 3-\frac{1}{3 n}}}{n+1}-\frac{n-1}{2 n(n+1)}-x$;

(4). $V_{n}^{* *}\left((t-x)^{2}, x\right)=2 x^{2}-\frac{x}{n}-2 x u_{n}(x)$;

(3). $V_{n}^{* *}\left((t-x)^{2}, x\right) \leq \frac{\varphi^{2}(x)}{n}-\frac{1}{2 n^{2}}$.

From Lemma 1, 2 and with the Korovkin-type property, we have the following convergence theorem.

Theorem 2. Let $f \in C_{\beta}[0,+\infty), x \geq \frac{\sqrt{3}}{3}$, we have $\lim _{n \rightarrow \infty} V_{n}^{* *}(f, x)=f(x)$.

\section{BETTER ERROR ESTIMATION}

Theorem 3. Let $f \in C_{B}[0,+\infty), x \geq \frac{\sqrt{3}}{3}, n \in N$, we have

$$
\left|V_{n}^{* *}(f, x)-f(x)\right| \leq 2 \omega\left(f, \delta_{n, x}\right),
$$

where $\delta_{n, x}=\sqrt{\frac{\varphi^{2}(x)}{n}-\frac{1}{2 n^{2}}}$. 
Proof. Let $f \in C_{B}[0,+\infty)$ and $x \geq 0$, using linearity and monotonicity of the operators $V_{n}^{* *}$, for every $\delta>0, n \in N$, we get

$$
\left|V_{n}^{* *}(f, x)-f(x)\right| \leq \omega(f, \delta)\left(1+\frac{1}{\delta} \sqrt{V_{n}^{* *}\left((t-x)^{2}, x\right)}\right) .
$$

Applying Lemma 2 and choosing $\delta=\delta_{n, x}$, the proof is complete.

Remark 3.1. (1).[8] For the Baskakov-Kantorovich operators given by (1.1), we may write that, for $f \in C_{B}[0,+\infty)$ and $x \geq 0, n \in N$,

$$
\left|V_{n}^{*}(f, x)-f(x)\right| \leq 2 \omega\left(f, \alpha_{n, x}\right),
$$

where $\alpha_{n, x}=\sqrt{\frac{x(1+x)}{n}+\frac{1}{3 n^{2}}}$.

(2).We can see that the error estimation in Theorem 3 is better than that of (3.1) provided $f \in C_{B}[0,+\infty), x \geq \frac{\sqrt{3}}{3}$.

Indeed, it is clear that

$$
\frac{x(1+x)}{n}-\frac{1}{2 n^{2}}<\frac{x(1+x)}{n}+\frac{1}{3 n^{2}},
$$

which guarantees that $\delta_{n, x}<\alpha_{n, x}$ for $x \geq \frac{\sqrt{3}}{3}$.

We say that a bounded function $f \in C[0,+\infty)$ belongs to $\operatorname{Lip}_{M}(\alpha)$ if the inequality $|f(t)-f(x)| \leq M|t-x|^{\alpha}$ holds for all $t \in[0,+\infty)$.

Theorem 4. For every $f \in \operatorname{Lip}_{M}(\alpha), x \geq \frac{\sqrt{3}}{3}$ and $n \in N$, we have

$$
\left|V_{n}^{* *}(f, x)-f(x)\right| \leq M\left\{\frac{\varphi^{2}(x)}{n}-\frac{1}{2 n^{2}}\right\}^{\frac{\alpha}{2}} .
$$

Proof. Since $f \in \operatorname{Lip}_{M}(\alpha), x \geq 0$, using the Hölder inequality with $p=\frac{2}{\alpha}, q=$ $\frac{2}{2-\alpha}$, we have

$$
\begin{aligned}
& \left|V_{n}^{* *}(f, x)-f(x)\right| \leq V_{n}^{* *}(|f(t)-f(x)|, x) \leq M V_{n}^{* *}\left(|t-x|^{\alpha}, x\right) \\
& \leq M\left(V_{n}^{* *}\left(|t-x|^{2}, x\right)\right)^{\frac{\alpha}{2}} \leq M\left\{\frac{\varphi^{2}(x)}{n}-\frac{1}{2 n^{2}}\right\}^{\frac{\alpha}{2}} .
\end{aligned}
$$

Remark 3.2. The classical Baskakov-Kantorovich operators given by (1.1) satisfy

$$
\left|V_{n}^{*}(f, x)-f(x)\right| \leq M\left\{\frac{x(1+x)}{n}+\frac{1}{3 n^{2}}\right\}^{\frac{\alpha}{2}},
$$

respectively for $f \in \operatorname{Lip}_{M}(\alpha), x \geq \frac{\sqrt{3}}{3}$ and $n \in N$.

It follows from (3.2) that the rate of convergence of the operators $V_{n}^{* *}$ for the Lipschitz class functions is better than the error estimation given by (3.3) whenever $x \geq \frac{\sqrt{3}}{3}$. 


\section{A Voronovskaya-type Theorem}

Along the same lines of the proof of Theorem 4.2 in [1], we have a Voronovskayatype theorem for the operators $V_{n}^{* *}$ given by (1.2).

Theorem 5. For every $f \in C_{\beta}[0,+\infty)$ such that $f^{\prime}, f^{\prime \prime} \in C_{\beta}[0,+\infty)$, we have

$$
\lim _{n \rightarrow \infty} n\left\{V_{n}^{* *}(f, x)-f(x)\right\}=-\frac{1}{2} f^{\prime}(x)+\frac{\varphi^{2}(x)}{2} f^{\prime \prime}(x)
$$

uniformly with respect to $x \in\left[\frac{\sqrt{3}}{3}, b\right]\left(b>\frac{\sqrt{3}}{3}\right)$.

Acknowledgements. This work was partially supported by the NSF of Hebei Province(A2012205028) and NSF of Hebei Normal University (L2010Z02).

\section{REFERENCES}

[1] O. Duman, M. A. Özarslan and B. D. Vecchia, Modified Szász-Mirakjan-Kantorovich operators preserving linear functions, Turk J. Math., 33, (2009) 151-158.

[2] J. P. King, Positive linear operators which preserve $x^{2}$, Acta Math. Hungarica, 99, (2003) 203-208.

[3] O. Agratini, Linear operators that preserve some test functions,Int. J. Math. Math. Sci., Art. ID 94136, (2006) 1-11.

[4] O. Agratini, On the iterates of a class of summation-type linear positive operators, Comput. Math. Appl., 55, (2008) 1178-1180.

[5] H. Gonska, P. Piţul and I. Raşa, General King-type operators, Result. Math., 53, (2009) 279-286.

[6] N. I. Mahmudov, Korovskin-type theorems and applications, Cent. Eur. J. Math., 7, (2009) 348-356.

[7] L. Rempulska, K. Tomczak, Approximation by certain linear operators preserving $x^{2}$, Turk. J. Math., 33, (2009) 273-281.

[8] Zhanjie Song, The Pointwise Estimate for Three Kinds of Baskakov-type Operators, Southeast Asian Bulletin of Mathematics, 28, (2004) 561-571.

College of Mathematics and Information Science,

Hebei Normal University, Shijiazhuang 050024, P.R.China

E-mail address: qiqiulan@163.com, yanggeshida@163.com 